\title{
On the Signal Filtering Property of CT Incremental Sigma-Delta ADCs
}

\author{
Johannes Wagner, Patrick Vogelmann and Maurits Ortmanns \\ Institute of Microelectronics, University of Ulm, D-89081 Ulm, Germany \\ Email: johannes.wagner@uni-ulm.de
}

\begin{abstract}
In this paper the calculation of the signal behavior and the achievable signal-to-quantization-noise ratio of continuous-time (CT) incremental Sigma-Delta (I-SD) ADCs is described. The presented method allows for the analysis of I-SD ADCs in frequency domain including the specific non-idealities of the CT modulator. In the state of the art, it is described that the omission of the preceding sample-and-hold for the I-SD ADC alters the transfer characteristic compared to a Nyquistrate converter. So far, for CT I-SD ADCs this behavior is only investigated via simulations. In this work the model of a discretetime I-SD ADC is generalized and adapted for the CT case. This allows to analytically obtain the signal and noise transfer function of the I-SD ADC in frequency domain in combination with arbitrary reconstruction filters due to the utilization of the lifting method in a fast and accurate way.
\end{abstract}

Index Terms-Incremental, Sigma-Delta, transfer function.

\section{INTRODUCTION}

The advances in CMOS scaling over the last decades allowed digital circuits to improve enormously, making the signal processing in the digital domain very efficient due to higher transit frequencies and the down-scaling of the transistors. The therefore ongoing shift to the digital domain raises a high demand on high performance analog-to-digital converters (ADCs). Analog circuits, however, do not equally benefit from technology scaling, thus classically used ADC architectures become more difficult to design. By trading in circuit precision against time, one type of ADC living with this trend is the Sigma-Delta (SD) ADC, which is based on oversampling and noise shaping [1]. However, its averaging behavior excludes it from being used in applications where sample-to-sample conversion is required, such as in multiplexed or other Nyquist-rate applications.

A solution for this drawback can be the usage of an incremental Sigma-Delta ADC [2]. It provides the benefits of both worlds, the Nyquist-rate behavior and the oversampling and noise shaping capabilities of a freely running SD ADC. The I-SD ADC, consisting of an incremental Sigma-Delta modulator (I-SDM) and a reconstruction filter with subsequent down-sampling, is depicted schematically in Fig. 1a. The working principle of the I-SD ADC is similar to a conventional Sigma-Delta ADC. The difference is the usage of a reset after $M$ cycles at the internal sampling rate $f_{\mathrm{s}}$ as in Fig. $1 \mathrm{~b}$. This reset window removes any memory from the system leading to true Nyquist-rate behavior [3]. As in their freely running counterparts, the I-SDM in Fig. 1a can thereby be realized with a discrete-time (DT) or CT loopfilter implementation.

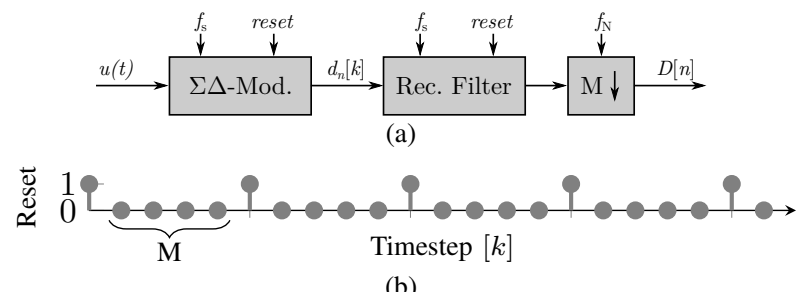

(b)

Fig. 1. Basic diagram of an I-SD ADC (a) and its reset signal (b).

Usually, a sample-and-hold element $(\mathrm{S} \& \mathrm{H})$ precedes the ISDM. As a consequence, the I-SD ADC has a constant signal transfer function (STF) equal to one. A preceding anti-aliasing filter (AAF) has to guarantee the Nyquist criterion. However, it can be omitted as proposed in [4] or [5], as the applied input signal is usually only slowly varying as compared to the internal sampling rate $f_{\mathrm{s}}$. This leads to a modified transfer characteristic with regard to the input signal, showing lowpass characteristic with a slight signal attenuation at and above the edge of the signal band. Throughout this paper, the absence of the $\mathrm{S} \& \mathrm{H}$ is assumed. For individual DT I-SD ADCs as e.g. in [6] or [7], the altered transfer behavior is analyzed analytically. However, for the CT case, this effect is only mentioned e.g. in [4] and determined by simulations, but has so far not been generally analyzed.

In this work, a general way to calculate the STF of CT ISD ADCs utilizing arbitrary reconstruction filters is presented, which has, to the authors knowledge, not been covered in literature so far. It makes use of the lifting method [8] and is able to directly include non-idealities as excess-loop-delay (ELD), finite DC gain and gain-bandwidth-product (GBW) into the calculation. To achieve this, the principles for calculating the transfer-behavior of DT I-SD ADCs are reviewed and subsequently extended and adapted to CT domain. Moreover, the same method allows to derive a noise transfer function (NTF) for I-SD ADCs. Based on the calculated NTF, an estimate of the signal-to-quantization-noise ratio (SQNR) can be obtained in frequency domain. This is especially useful in an early design phase on system-level.

Section II discusses the working principle of I-SD ADCs. The transfer behavior of DT I-SD ADCs is outlined in Section III. In Section IV an overview of the lifting method is given. Subsequently, the work from Section III is extended to CT I-SD ADCs and the SQNR estimation is introduced. In Section V simulations on system-level verify the applicability of the presented method. Section VI concludes the paper. 


\section{I-SD ADC WORKING PRINCIPLE}

A block-diagram of the basic architecture is shown in Fig. 1a, consisting of the I-SDM, the digital reconstruction and decimation filter. As an example for further explanations, the linear model of a first order DT I-SD ADC is taken as shown in Fig. 2a. Therefore, the quantizer is replaced by a noise source $e[k]$ and the linear gain $q$ as e.g. described in [1]. In contrast to the frequency domain analysis of conventional Sigma-Delta modulators (SDMs), DT I-SD ADCs are often described in time domain. Due to the finite number of time steps, the equations can be determined in a straightforward way. In the case of a DT I-SDM the continuously running input signal $u(t)$ is sampled by the switched capacitor network at the integrator input resulting in samples $u_{n}[k]$. Here $n$ denotes the $n$-th Nyquist rate sample and $k=1 \ldots M$ is the running variable of the oversampled modulator, where $M$ samples at $f_{\mathrm{s}}$ are used to generate one Nyquist sample at $f_{\mathrm{N}}$. Also in I-SDM, $M$ is often called the oversampling ratio (OSR). Consequently the integrator output is calculated in time domain by

$$
y_{n}[k]=\sum_{l=1}^{k}\left(u_{n}[l]-d_{n}[l]\right) .
$$

The basic difference of an I-SDM to a conventional SDM is its periodically applied reset window as shown in Fig. 1b. Therefore, the I-SDM generates $M$ samples $d_{n}[k]$ for $k \in$ $[1, M]$ with

$$
d_{n}[k]=q \cdot y_{n}[k]+e[k]
$$

which the reconstruction filter and the subsequent decimator use to create one sample

$$
D[n]=\frac{1}{M} \sum_{l=1}^{M} d_{n}[l] .
$$

The output $D[n]$ yields the digital representation of the analog input at Nyquist-rate $f_{\mathrm{N}}$. Therefore, each conversion cycle is independent from each other and true Nyquist ADC behavior is obtained. A detailed description can be found in [1] or [3].

\section{TRANSFER ChARACTERISTIC OF DT I-SD ADCS}

The STF or NTF of a conventional DT SDM are usually obtained with the help of the Z-transform by using the linearized, time invariant system model. However, in the overall transfer characteristic for I-SD ADCs also the reconstruction filter and the time-variant behavior due to the reset have to be considered. In Section II, it is shown that DT I-SD ADCs can be described in time domain in a straightforward way. Nonetheless, a description via the Z-transform or in frequency domain is often desired. Therefore, in this chapter the representation of an I-SD ADC as filter with finite impulse response (FIR) as introduced in [7] is shortly reviewed. Afterwards, this work extends that approach to higher order modulators and shows the calculation of the overall STF and NTF via time domain considerations and in frequency domain for DT and CT modulator implementations. In the following a normalized sampling frequency $f_{\mathrm{s}}=1$ is assumed for simplicity and readability of the analysis.

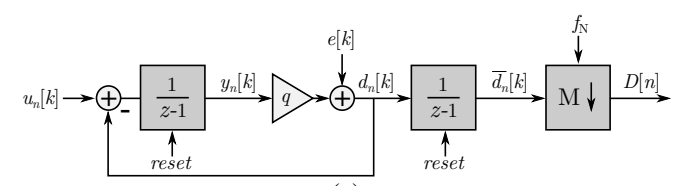

(a)

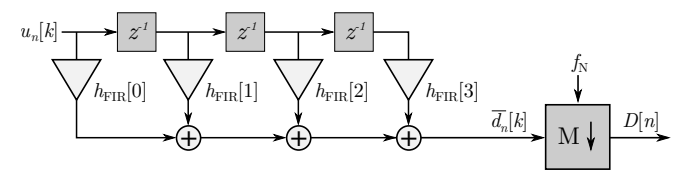

(b)

Fig. 2. Linear model of a DT first order I-SD ADC (a) and equivalent FIR filter with an oversampling ratio of $M=3$.

\section{A. I-SD ADC as FIR Filter}

In this section it is shortly reviewed that DT I-SD ADCs can be modeled as an FIR system as it was introduced in [7] for a first order DT modulator. The reset changes the system from an LTI to a linear time varying system (LTV). The idea is shown for a general DT transfer function with applied reset and can therefore be the basis for both, the NTF and STF calculation. The Z-transform of the overall transfer function of a freely running DT Sigma-Delta ADC, $T F_{\text {free, }}$, is given by

$$
T F_{\text {free }}(z)=T F_{\mathrm{sdm}}(z) \cdot H_{\text {rec }}(z)
$$

with $T F_{\mathrm{sdm}}(z)$ being the STF or NTF of the freely running SDM and $H_{\text {rec }}(z)$ being the transfer function of its reconstruction filter. In time domain, this yields a convolution sum

$$
h_{\text {free }}[k]=h_{\mathrm{sdm}}[k] * h_{\text {rec }}[k]=\sum_{l=-\infty}^{\infty} h_{\mathrm{sdm}}[k-l] \cdot h_{\mathrm{rec}}[k]
$$

with the respective impulse responses of the two subsequent systems. Without reset, the impulse response $h_{\text {free }}[n]$ of the ADC would be infinite due to the infinite impulse response $h_{\mathrm{sdm}}[n]$ of the modulator. However, it is proven in [7] that by truncating the convolution sum (5), the impulse response of an equivalent FIR filter

$$
h_{\mathrm{FIR}}[k]=\sum_{l=0}^{M-1} h_{\mathrm{sdm}}[k-l] \cdot h_{\mathrm{rec}}[k]
$$

can be obtained for $k \in[0, M-1]$, which accounts for the reset, as it is the case in an I-SD ADC.

\section{B. Generalization of the TF Calculation}

In [7] a first order DT I-SD ADC was described, however, this approach can be generalized to higher order modulators.

1) Calculation via Time Domain Operations: By generalizing the mathematical model of the DT I-SD ADC as an FIR filter, (6) can be rewritten as

$$
\begin{aligned}
h_{\mathrm{FIR}}[k] & =h_{\text {free }}[k] \cdot \operatorname{rect}_{M}[k] \\
& =\mathcal{Z}^{-1}\left\{T F_{\text {free }}(z)\right\}[k] \cdot \operatorname{rect}_{M}[k] .
\end{aligned}
$$

The expression $\operatorname{rect}_{M}[k]$ is a DT rectangular window function with length $M$

$$
\operatorname{rect}_{M}[k]= \begin{cases}1, & 0 \leq k \leq M-1 \\ 0, & \text { otherwise }\end{cases}
$$




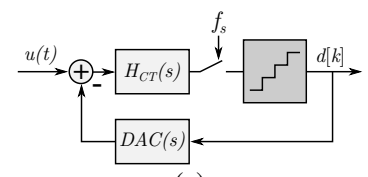

(a)

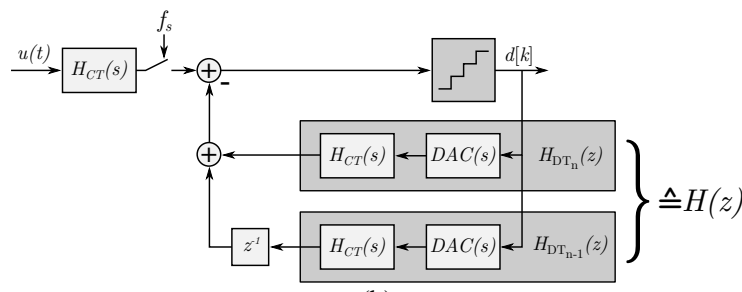

(b)

Fig. 3. Block diagram of a CT SDM (a) and the corresponding lifted mode (b) as basis for the STF calculation.

and $T F_{\text {free }}(z)$ is the combination of the STF or NTF of the freely running SDM and the reconstruction filter transfer function as defined in (4). As commonly known, the impulse response of an FIR are the weighting coefficients of the filter taps. Therefore, the Z-domain representation can be written as

$$
T F_{\mathrm{dt}, \text { incr }}(z)=\sum_{l=0}^{M-1} h_{\mathrm{FIR}}[l] \cdot z^{-l} .
$$

Consequently, by utilizing (7) and (9), the Z-domain representation of any modulator transfer function $T F_{\mathrm{sdm}}(z)$ and any reconstruction filter transfer functions $H_{\text {rec }}(z)$ can be obtained. This approach is applicable to both STF and NTF. With the application of the discrete-time Fourier transform (DTFT) and by substituting $z$ by $z=e^{j 2 \pi f}$, the frequency response can be obtained:

$$
T F_{\mathrm{dt}, \text { incr }}(f)=\sum_{l=0}^{M-1} \mathcal{Z}^{-1}\left\{T F_{\text {free }}(z)\right\}[l] \cdot e^{-j 2 \pi f l}
$$

2) Calculation in Frequency Domain: Above, the calculation of the I-SD ADCs transfer function is described via the impulse response in the time domain. However, so far literature has not shown the calculation directly in the frequency domain, which might be useful, if the Z-domain representation is not entirely known due to e.g. non-ideal behavior. This can be achieved by applying the DTFT directly to (7) yielding

$$
T F_{\mathrm{dt}, \text { incr }}(f)=T F_{\mathrm{dt}, \text { free }}(f) \circledast X_{\text {rect }, N}(f)
$$

with

$$
\begin{aligned}
X_{\text {rect }, M}(f) & =\operatorname{DTFT}\left\{\operatorname{rect}_{M}[k]\right\} \\
& =\frac{\sin (M \pi f)}{\sin (\pi f)} \cdot e^{-j(M-1) \pi f} .
\end{aligned}
$$

The necessary $\circledast$-operator stands for the CT circular convolution which requires the solution of the convolution integral $X(f) \circledast Y(f)=\int_{f_{o}}^{f_{o}+f_{\mathrm{s}}} X(\nu) \cdot Y(f-\nu) d \nu$.

\section{TRANSFER CHARACTERISTIC OF CT I-SD ADCS}

CT I-SD ADCs have not been proposed often in the literature. Still, they were always emphasized to feature similar transfer function properties as their freely running counterparts, such as an implicit AAF. Nonetheless, the calculation of the frequency response of CT I-SD ADCs is, to the best of the authors knowledge, not covered in literature so far. This section presents a method to obtain the frequency response of a CT I-SD ADC having the possibility to include non-ideal effects, which originate from in the CT I-SDM.

\section{A. Lifting Method for Freely Running SDMs}

There have been several methods proposed dealing with the calculation of the transfer function of freely running CT SDMs [9][10]. These methods share the common idea that the feedback path around the quantizer of the CT SDM can be investigated as an equivalent DT filter, due to its sampled input and output nature. The system is therefore modeled as a CT prefilter accounting for the CT behavior and a DT filter in the feedback accounting for the DT behavior. This idea still holds true as basis for further considerations on CT ISD ADCs. Nonetheless, the methods to obtain the equivalent DT filter - as described in the mentioned papers - are not generally applicable. The transformation method in [9] cannot account for excess-loop-delay (ELD) and the approach in [10] requires the spectral representation of the feedback DAC.

In order to overcome the limitations of these transformations, the lifting method is used in this work to obtain the equivalent DT filter [8]. The DT model, analytically derived with this method, behaves exactly as the CT model at the sampling instants. Therefore, it is also an accurate method to simulate a CT modulator with the high speed solvers of its DT counterpart. Finally, in [11] it was shown that the lifting method can be utilized to obtain the NTF and STF of freely running CT SDMs including arbitrary DAC feedback waveforms and non-ideal loopfilter behavior - which is shortly reviewed in the following as reference.

An arbitrary CT SDM as in Fig. 3a with the CT filter function $H_{C T}(s)$ and the transfer characteristic of the feedback DACs can be transformed by the lifting method to a model with CT prefilter $H_{C T}(s)$ and the DT filter $H(z)$ in the feedback path as in Fig. 3a, which is the conventional way of the STF calculation of a freely running CT SDM proposed in [9]. The DT filter is obtained by

$$
H(z)=H_{\mathrm{DT}_{n}}(z)-H_{\mathrm{DT}_{n-1}}(z) \cdot z^{-1} .
$$

The function $H_{\mathrm{DT}_{n}}(z)$ accounts for the non-delayed signal part in the current clock cycle, whereas the delayed function $H_{\mathrm{DT}_{n-1}}(z)$ accounts for the signal part that is shifted into the next clock cycle by ELD. A more detailed description of how lifting is used to calculate the STF on non-ideal CT loopfilters can be found in [11]. With the equivalent DT filter $H(z)$ in (13), the NTF of the freely running CT modulator can be expressed by

$$
N T F_{\mathrm{ct}, \mathrm{sdm}}(z)=\frac{1}{1+q \cdot H(z)}
$$

in Z-domain and transformed to frequency domain with the usage of the DTFT by substituting $z=\mathrm{e}^{j 2 \pi f}$. As a result, the STF of the CT SDM including non-idealities can then be 
written corresponding to [11] by concatenating the prefilter $H_{\mathrm{CT}}(j 2 \pi f)$ and the NTF accordingly:

$$
S T F_{\mathrm{ct}, \mathrm{sdm}}(f)=\frac{q \cdot H_{\mathrm{CT}}(j 2 \pi f)}{1-q \cdot H\left(\mathrm{e}^{j 2 \pi f}\right)} .
$$

\section{B. Calculation of NTF for CT I-SD ADC}

The NTF of an I-SD ADC allows to directly estimate the SQNR of the I-SD ADCs in combination with the white noise model in a general way, comparable to its freely running counterpart. The estimated SQNR is determined by

$$
S Q N R_{\text {est }}=\frac{\Delta^{2}}{12} \cdot \int_{-0.5}^{0.5}\left|N T F_{\text {incr }}(f)\right|^{2} d f
$$

with the quantizer step size $\Delta$ [1]. As the NTF of the freely running CT SDM can be expressed due to the lifting method in Z-domain by means of (14), the approach from Section III-B1 can be applied to obtain the NTF of an CT I-SD ADC. Inserting (14) in (7) and the resulting term in (9) gives

$$
N T F_{\mathrm{ct}, \text { incr }}(f)=\sum_{l=0}^{M} \mathcal{Z}^{-1}\left\{N T F_{\mathrm{ct}, \text { free }}(z)\right\}[l] \cdot e^{-j 2 \pi l f}
$$

with the combined transfer function of the freely running modulator and reconstruction filter $N T F_{\mathrm{ct}, \text { free }}(z)=$ $N T F_{\mathrm{ct}, \mathrm{sdm}}(z) \cdot H_{\text {rec }}(z)$ as in (4).

\section{Calculation of STF for CT I-SD ADC}

For the CT modulator, the reset window is also continuoustime and can be expressed by the rectangular window function

$$
\operatorname{rect}_{T}(t)= \begin{cases}1, & |t|<\frac{T_{\mathrm{M}}}{2} \\ 0, & |t|>\frac{T_{\mathrm{M}}}{2}\end{cases}
$$

with $T_{\mathrm{M}}=\frac{M}{f_{\mathrm{s}}}$. However, due to the mixed-signal nature of the modulator, time-domain calculations are not as straightforward as shown for the DT modulators in the method in Section III-B1. A feasible way to calculate the STF is in frequency domain. Therefore, (18) is transformed via the Fourier transform to frequency domain, similarly as in Section III-B2:

$$
X_{\text {rect }, T}(f)=\mathcal{F}\left\{\operatorname{rect}_{T}(t)\right\}=\operatorname{sinc}(f)=\frac{\sin \left(\pi \cdot f / f_{\mathrm{s}}\right)}{\pi \cdot f / f_{\mathrm{s}}}
$$

For a causal system, the rectangular function (18) needs to be shifted by $T_{\mathrm{M}} / 2$ and therefore the reset window in the frequency domain becomes

$$
X_{\text {rect }, T}^{\prime}(f)=\operatorname{sinc}(f) \cdot e^{-j T \pi f / f_{\mathrm{s}}} .
$$

The frequency response of $X_{\text {rect, } T}^{\prime}(f)$ is illustrated alongside its DT counterpart in Fig. 4, visualizing its CT nature as the amplitude decreases with increasing frequency. In contrast, the frequency response of the DT rectangular function is expectedly mirrored at $0.5 f_{\mathrm{s}}$. Consequently, the multiplication with the reset window in the time domain corresponds to a convolution of the combined transfer function of the freely running modulator and the reconstruction filter $S T F_{\text {ct,free }}(f)=S T F_{\text {ct,sdm }}(f) \cdot H_{\text {rec }}\left(e^{j 2 \pi f}\right)$ with the frequency response of the $\mathrm{CT}$ reset window:

$$
S T F_{\mathrm{ct}, \text { incr }}(f)=S T F_{\mathrm{ct}, \text { free }}(f) * X_{\text {rect }, T}^{\prime}(f) .
$$

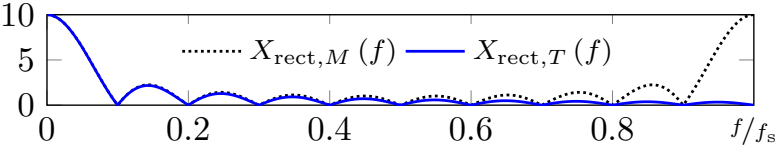

Fig. 4. Fourier transform of DT and CT rectangular functions for $M=10$.

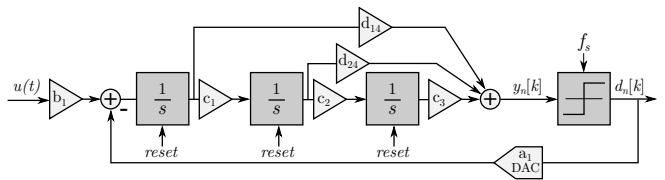

(a)

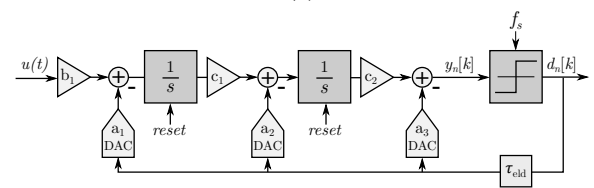

(b)

Fig. 5. Block diagram of a CT $3^{\text {rd }}$ order I-SDM in CIFF architecture (a) and a CT $2^{\text {nd }}$ order I-SDM in CIFB architecture.

\section{PROOF OF CONCEPT}

In order to proof the validity of the presented analysis, in this section the accuracy of the calculated transfer functions is investigated on system-level. Alongside two exemplary CT ISD ADCs, two DT I-SD ADCs are shown in order to compare the different STFs and NTFs. Non-idealities as e.g. finite GBW or DC gain are not covered due to space limitations. However, simulations revealed equally good matching.

\section{A. CoI Reconstruction Filter}

The presented approach can be used for I-SD ADCs comprising arbitrary, linear reconstruction filters. In the presented examples, a chain-of-integrator $(\mathrm{CoI})$ reconstruction filter is used, which has the same reset window applied. The CoI filter is utilized as it is commonly used in I-SD ADCs due to its simplicity [1]. For a first order I-SD ADC, this can be as simple as in (3). In general, the non-delaying, $L$-th order filter can be expressed in Z-domain by

$$
H_{r e c},(z)=\sum_{l=1}^{M} w_{\mathrm{L}}[l] \cdot z^{-(l-1)}
$$

with the filter weights

$$
w_{\mathrm{L}}[l]=\frac{1}{(L-1) !} \prod_{i=1}^{L-1}(M-l+i) .
$$

\section{B. Comparison of Calculations and Simulations}

The architectures of the exemplary I-SDMs are shown in Fig. 5. A $3^{\text {rd }}$ order CT I-SDM in CIFF architecture is depicted in Fig. 5a and a $2^{\text {nd }}$ order modulator in CIFB architecture in Fig. 5b. The DT counterparts reassemble these examples with DT integrators. The $2^{\text {nd }}$ order CT modulator has an additional compensation path for an ELD of $0.5 f_{\mathrm{s}}$, which is omitted in the DT case. All integrator outputs are scaled to full scale swing and NRZ DACs are used. The OSR is chosen as $M=150$. The simulations use an input signal at $0.3 f_{\mathrm{b}}$ and $-6 \mathrm{dBFS}$ amplitude and are based on $2^{14}$ samples at $f_{\mathrm{N}}$. 
TABLE I

COEFFICIENTS AND QUANTIZER GAIN OF THE EXAMPLE DESIGNS.

\begin{tabular}{c|cccccccc|c}
\hline & $\mathrm{b}_{1} / \mathrm{a}_{1}$ & $\mathrm{a}_{2}$ & $\mathrm{a}_{3}$ & $\mathrm{~d}_{14}$ & $\mathrm{~d}_{24}$ & $\mathrm{c}_{1}$ & $\mathrm{c}_{2}$ & $\mathrm{c}_{3}$ & $q$ \\
\hline $\mathrm{CT}_{3}$ & 0.188 & - & - & 5.316 & 6.807 & 0.424 & 0.178 & 6.016 & 1.000 \\
$\mathrm{CT}_{2}$ & 0.040 & 0.250 & 0.165 & - & - & 2.114 & 1 & - & 4.279 \\
$\mathrm{DT}_{3}$ & 0.188 & - & - & 6.835 & 7.879 & 0.424 & 0.178 & 6.016 & 1.001 \\
$\mathrm{DT}_{2}$ & 0.125 & 0.234 & - & - & - & 0.718 & 1 & - & 5.842 \\
\hline
\end{tabular}

TABLE II

SQNR OF THE EXEMPLARY ADCS AT -6 DBFS INPUT AMPLITUDE.

\begin{tabular}{lcccc}
\hline & $\mathrm{CT}_{2}$ & $\mathrm{CT}_{3}$ & $\mathrm{DT}_{2}$ & $\mathrm{DT}_{3}$ \\
\hline Estimated: & $73.6 \mathrm{~dB}$ & $99.4 \mathrm{~dB}$ & $75.2 \mathrm{~dB}$ & $99.4 \mathrm{~dB}$ \\
Simulated: & $74.8 \mathrm{~dB}$ & $100.9 \mathrm{~dB}$ & $75.5 \mathrm{~dB}$ & $100.2 \mathrm{~dB}$ \\
\hline
\end{tabular}

The coefficients of the modulators are shown in Table I for a single-bit quantizer. Alongside, the quantizer gain, obtained by simulations as described in [1], is stated.

1) STF: The STF of the DT I-SD ADC is numerically calculated based on (10), the CT STF based on (20). Simulations to proof the validity of the calculations are marked alongside the calculation results with crosses in Fig. 6 for the CT ADC and in Fig. 7 for the DT ADCs. The simulations match perfectly the calculated functions. The DT STFs in Fig. 7 clearly show the symmetry around $0.5 f_{\mathrm{s}}$ in contrast to the CT STFs with their continuous roll-off at higher frequencies. The lowered STF at higher frequencies of the second order I-SD ADCs becomes clearly visible as well. This is reasoned in the transfer characteristic of the second order CoI filter, which shows higher attenuation at high frequencies then the third order one.

2) NTF: The NTF of the CT I-SD ADC is numerically obtained based on (17) and the DT NTF based on (10). They are depicted in Fig. 6 for the CT case and in Fig. 7 for the DT case. The raised NTF of the second order modulators can be directly seen. Moreover, the calculated NTFs allow to directly estimate the SQNR of the I-SD ADC by (16) with the quantizer step size $\Delta=2$ in the case of the single-bit quantizer [1]. In Table II, the estimated and simulated SQNR values at the same input amplitude are stated. The respective values match closely. In order to verify the course of the NTF, the linearized model of the modulator is utilized for simulations. Therefor, the quantizer is replaced by its gain $q$ and the signal source representing the quantization noise as in Fig. 2a. The resulting values annotated in Fig. 6 and in Fig. 7 match again exactly with the calculations.

\section{CONCLUSION}

In this paper the calculation of transfer functions of CT I-SD $A D C s$ without $\mathrm{S} \& \mathrm{H}$ is presented. The mathematical derivation, which is based on the lifting method, can be used to obtain the transfer functions involving arbitrary reconstruction filters. Moreover, for the CT I-SD ADCs the non-idealities of the CT modulator as e.g. ELD can be accounted in the calculation. Therefore, the method to derive the transfer functions of a DT I-SD ADC is adapted to the NTF and extended for the CT nature of the STF. The given examples illustrate that the presented method allows a fast and precise way of calculating the transfer characteristics which match reference simulations. Moreover, the NTF allows to predict the SQNR of the ADC.

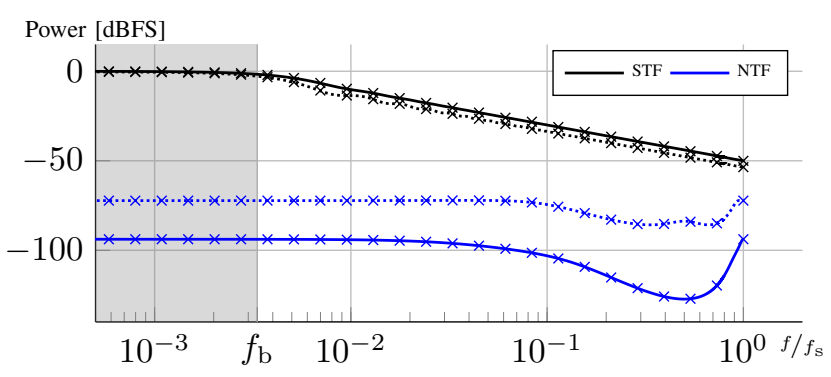

Fig. 6. Calculated STF and NTF of the exemplary $3^{\text {rd }}$ (solid) and $2^{\text {nd }}$ (dotted) order CT I-SD ADCs with annotated simulation results.

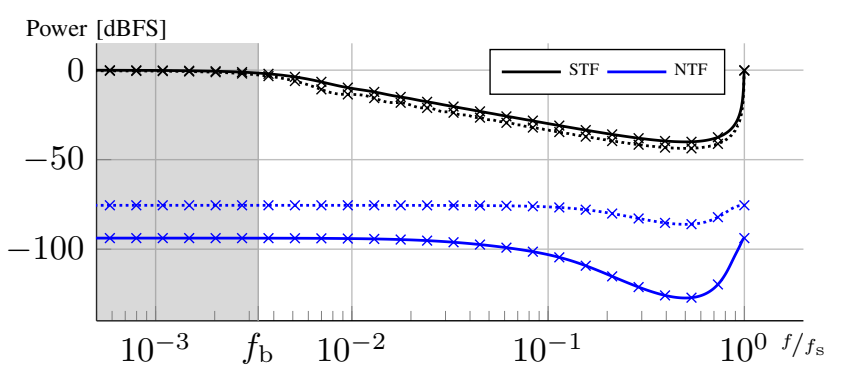

Fig. 7. Calculated STF and NTF of the exemplary $3^{\text {rd }}$ (solid) and $2^{\text {nd }}$ (dotted) order DT I-SD ADCs with annotated simulation results.

\section{ACKNOWLEDGMENT}

This work was supported by German Research Foundation under DFG grant OR 245/10-1.

\section{REFERENCES}

[1] S. Pavan, R. Schreier, and G. C. Temes, Understanding delta-sigma data converters. John Wiley \& Sons, 2017.

[2] J. Robert, G. C. Temes, V. Valencic, R. Dessoulavy, and P. Deval, "A 16-bit low-voltage CMOS A/D converter," IEEE Journal of Solid-State Circuits, vol. 22, no. 2, pp. 157-163, Apr 1987.

[3] J. Markus, J. Silva, and G. C. Temes, "Theory and applications of incremental $\Delta \Sigma$ converters," IEEE Transactions on Circuits and Systems I: Regular Papers, vol. 51, no. 4, pp. 678-690, Apr. 2004.

[4] S. Tao and A. Rusu, "A power-efficient continuous-time incremental sigma-delta adc for neural recording systems," IEEE Transactions on Circuits and Systems I: Regular Papers, vol. 62, no. 6, pp. 1489-1498, June 2015.

[5] P. Vogelmann, J. Wagner, A. Hänsler, and M. Ortmanns, "Improved SQNR and MSA in incremental $\Delta \Sigma$ modulators by using a recuperation phase," IEEE Transactions on Circuits and Systems II: Express Briefs, vol. 65, no. 5, pp. 557-561, May 2018.

[6] T. C. Caldwell and D. A. Johns, "Incremental data converters at low oversampling ratios," IEEE Transactions on Circuits and Systems I: Regular Papers, vol. 57, no. 7, pp. 1525-1537, Jul. 2010.

[7] S. Bannwarth, A. Wenzler, and W. Mathis, "On the transfer behaviour of incremental sigma delta converters," in Proc. VXV Int. Symp. Theoretical Engineering, Jun. 2009, pp. 1-5.

[8] M. Keller, A. Buhmann, M. Ortmanns, and Y. Manoli, "A method for the discrete-time simulation of continuous-time sigma-delta modulators," in 2007 IEEE International Symposium on Circuits and Systems, May 2007, pp. 241-244.

[9] R. Schreier and B. Zhang, "Delta-sigma modulators employing continuous-time circuitry," IEEE Transactions on Circuits and Systems I: Fundamental Theory and Applications, vol. 43, no. 4, pp. 324-332, Apr. 1996.

[10] S. Loeda, H. M. Reekie, and B. Mulgrew, "On the design of highperformance wide-band continuous-time sigma-delta converters using numerical optimization," IEEE Transactions on Circuits and Systems I: Regular Papers, vol. 53, no. 4, pp. 802-810, Apr. 2006.

[11] T. Brückner, M. Kiebler, M. Lorenz, C. Zorn, W. Mathis, and M. Ortmanns, "Calculating transfer functions of CT sigma-delta modulators with arbitrary DAC waveforms," in Proc. IEEE Int. Symp. Circuits and Systems (ISCAS2013), May 2013, pp. 1264-1267. 